\title{
On the Poincaré Group at the 5th Root of Unity
}

\author{
Marcelo Amaral • Klee Irwin
}

Received: date / Accepted: date

\begin{abstract}
Considering the predictions from the standard model of particle physics coupled with experimental results from particle accelerators, we discuss a scenario in which from the infinite possibilities in the Lie groups we use to describe particle physics, nature needs only the lower dimensional representations - an important phenomenology that we argue indicates nature is code theoretic. We show that the "quantum" deformation of the $S U(2)$ Lie group at the 5th root of unity can be used to address the quantum Lorentz group and gives the right low dimensional physical realistic spin quantum numbers confirmed by experiments. In this manner we can describe the spacetime symmetry content of relativistic quantum fields in accordance with the well known Wigner classification. Further connections of the 5th root of unity quantization with the mass quantum number associated with the Poincaré Group and the $S U(N)$ charge quantum numbers are discussed as well as their implication for quantum gravity.
\end{abstract}

Keywords Quantum Groups · Quantum Gravity · Quantum Information · Particle Physics · Quasicrystals · Fibonacci Anyons

\section{Introduction}

One of the key ideas of modern physics, which is present in the construction of the standard model of particle physics, is the concept of a field, which is a

\author{
Marcelo Amaral \\ Quantum Gravity Research \\ Los Angeles, $C A$ \\ E-mail: Marcelo@QuantumGravityResearch.org \\ Klee Irwin \\ Quantum Gravity Research \\ Los Angeles, $C A$ \\ E-mail: Klee@QuantumGravityResearch.org
}


representation of a Lie group. In this framework of quantum field theory, spin and mass arise via the representation theory of the Poincaré group. Charge is associated with internal gauge symmetry, the electric charge with the $U(1)$ Lie group and the color charge with $S U(3)$, for example. The reason as to why some group representations such as those associated with spacetime, i.e. the Poincaré group, are realized in nature and others are not is thus open for debate.

Physical principles inferred from observed phenomena serve as a roadmap in constructing fundamental physical theories. For example, the equivalence principle served as an important step in the development of general relativity; the uncertainty principle helped in the development of quantum mechanics; gauge symmetry principles and the principle of least action are important in the construction of quantum field theories; and the holographic principle is relevant in the context of string theory and the AdS/CFT correspondence [1, $2]$. The holographic principle is meaningful in this context as it stems from the open problem of quantizing spacetime and gravity along with the quest for unification of fundamental quantum fields. The holographic principle was proposed from logical considerations of physical phenomena associated with gravitational collapse and led to the conclusion that physics at the Planck scale is constrained to be lower dimensional and to possess finite degrees of freedom. Physics at the Planck scale can imply a violation and/or generalization of established principles like the uncertainty principle and Lorentz invariance [3-9]. In order to probe this ultimate scale of spacetime, new insights and fundamental principles must be realized [10-13].

In this context we discuss a quantizing principle for quantum gravity that is deeply correlated to the Lie algebraic basis of particle physics constrained by particle accelerator experiments, with the purpose of non-arbitrarily deriving our Planck scale code theoretic restrictions and degrees of freedom. We use the proposed code theoretic principle [14] to explain the phenomenological constraints in the representations of a Lie group. A physical spatiotemporal code is defined as: (1) a finite set of symbolic objects, (2) ordering rules and (3) syntactical freedom, (4) for the purpose of expressing meaning, i.e., self-referential physical meaning. In connection with the free will theorem [15] and strong free will theorem [16], the code theoretic principle states that nature is, at the fundamental level, a physical spatiotemporal code computing itself, which is a different ontological starting point from either randomness or determinism. The requirement of syntactical freedom for the purpose of expressing meaning brings the concept of free will choices within the code, a concept whose philosophical implications we will not discuss here. Instead, we will focus on aspects of representation theory of Lie groups connected with the relativistic quantum fields in the attempt to clarify the need for this new principle. A code theoretic physical principle, in agreement with results from the experimental physics of particle accelerators, should advance in lock-step with the existing body of fundamental physics derived from quantum information theory [17-22] - the digital physics paradigm, in particular - the idea that reality is numerical at its core [14,23-33]. The digital physics approach 
contemplates any numerical method of discretizing spacetime and action into a finite set of values - the core physical philosophy of generalized quantum theory extended to the regime of space and time [34]. In this paper, our postulate and assumptions are based on the digitalization or pixelization of physics and aim to bring new insights within this field.

In the context of representation theory of Lie groups, the code theoretic approach can be implemented by the "quantum" deformation of the $S U(2)$ Lie group at a root of unity [35]. This $S U(2)$ quantum group can be used as a building block in addressing quantum deformations of large dimensional Lie groups. We propose that when the deformation parameter is complex and a 5 th root of unity we have the desired conditions to recover particle physics phenomenology and fulfill the code theoretic principle.

This paper is organized as follows: in Section 2 we introduce the concept of physical codes with two practical examples. In Section 3 we discuss elements of the representation theory of the Poincaré group, suggesting the necessity of a code theoretic principle and its connections to quantum gravity, as well as the requirement for a consistent quantization of this group to impose restrictions on its representations. We present our conclusions in Section 4.

\section{Physical Codes}

The code theoretic concept appears in different fields such as computer science, information theory, genetics, mathematics and linguistics [36]. Any language is a code. Physical codes can be topological or geometrical. As mentioned in the introduction, a physical spatiotemporal code is: (1) a finite set of symbolic objects, (2) ordering rules and (3) syntactical freedom, (4) for the purpose of expressing meaning, i.e., self-referential physical meaning. Let us clarify the code theoretic concept with two examples, anyonic topological codes and quasicrystalline codes.

\subsection{Anyonic Topological Codes}

For three-dimensional quantum systems, the exchange of two identical particles may result in a sign change of the wave function which can be used to distinguish fermions from bosons. Two-dimensional quantum systems, on the other hand, have much richer quantum statistical behaviors. It is possible to assign an arbitrary phase factor, or even a whole unitary matrix, to the evolution of the wave function where the states are called anyons [37-39]. To describe a system of anyons, the usual approach is to list the species of anyons in the system, also called the particle types, topological charges or simply labels. These are the "letters" or the finite set of symbolic objects of this code - an anyonic topological code [37,38,40-42]. Then there are the so-called fusion rules $[37,38]$, which specify how these fundamental labels can be coupled. 
These rules are not deterministic and depending on the class of anyons ${ }^{1}$ we are dealing with, there can be various degrees of freedom, which implement the ordering rules and syntactical freedom ${ }^{2}$. The last component of a code is its function to express meaning, such as a specific quantum computation. It is well known that some anyonic systems like the simple non abelian anyon the Fibonacci anyon - are capable of universal quantum computation [37,38, 42]. Fibonacci anyon fusion rules are irreducibly simple and can be understood in terms of the representation theory of Hopf algebras and quantum groups - a generalization of Lie groups - especially the quantum $S U(2)^{3}$ [40]. The fusion rules for Fibonacci anyons are in this case:

$$
\begin{aligned}
& 1 \otimes 1=0 \oplus 1 \\
& 0 \otimes 1=1 \\
& 1 \otimes 0=1
\end{aligned}
$$

where we make use of the spin label to express the representations. For example, the coupling between spin 1 representations - our code letters - gives a spin 0 or a spin 1 representation. With this simple fusion rule and a small number of representations it is possible to express universal quantum computations. If such topological codes existed in nature and were easy to artificially create for technological applications to quantum computation, we should expect to see experimental evidence, like for example, both theoretical and experimental results reported in the literature of the fractional quantum hall effect $[37,38,44]$.

\subsection{Quasicrystalline Codes}

A quasicrystal is a structure that is ordered but not periodic. It has long-range quasiperiodic translational order and long-range orientational order. It has a finite number of prototiles or "letters" as its finite set of symbolic objects and it has a discrete diffraction pattern indicating order but not periodicity. An example of spatiotemporal codes naturally occurring in nature are quasicrystals such as DNA, which Schrödinger called aperiodic crystals [45], and various metallic quasicrystals [46-49]. Quasicrystalline codes are dynamic geometrical spatiotemporal codes based on the first principles of Euclidean projective geometry.

\footnotetext{
1 Note that the fusion rules for Fibonacci anyons we are discussing here seem similar to the fusion rules for the Ising model, which in turn, represent just one particular example of a rational conformal field theory $[41,43]$. The key property of these theories is that they have a finite number of primary fields when the representations of the infinite dimensional $2 \mathrm{D}$ conformal group are constructed out of the highest weight states labeled by rational values of the central charge (related to the highest weight vector). These theories can thus provide a large number of codes in the sense discussed here.

2 See equation (1) for the precise meaning of syntactical freedom which is expressed in the rule $1 \otimes 1=0 \oplus 1$.

3 See Section 3.1.
} 
Mathematically, there are three common ways of generating a quasicrystal: the cut-and-project method (projection of an irrational slice of a higher dimensional crystal) [46], the dual grid method [46], and the Fibonacci grid method [50]. Finite quasicrystals can be constructed by matching rules and it is interesting that a given set of local interactions or matching rules enforces a quasiperiodic ground state to express a physical object such as the metallic quasicrystals observed so far. Quasicrystals were discovered via synthesis in 1982 and first reported in 1984 [51]. Around 300 or so quasicrystals have been synthesized since then in addition to those found in nature. All of these quasicrystals can be understood as projections of higher dimensional lattices such as the pure mathematical four dimensional Elser-Sloane quasicrystal [52, $53]$, which is a cut-and-projection of the $E_{8}$ lattice. The simplest quasicrystals possible are the 1D class with only two letters or lengths, such as the two length Fibonacci chain $[46,47]$. The Penrose tiling, a 2D quasicrystal, is a network of $1 \mathrm{D}$ quasicrystals. 3D quasicrystals, such as a $3 \mathrm{D}$ Penrose tiling (Ammann tiling) are networks of 2D quasicrystals, which are each networks of 1D quasicrystals, mainly partially deflated Fibonacci chains that generate additional length based letters other than the primary two of the Fibonacci chain quasicrystal. Accordingly, the irreducible building blocks of all quasicrystals are 1D quasicrystals. Physically, the "letters" of these $1+n$ dimensional spatiotemporal codes can be seen as lengths between vacant or occupied energy wells. A 1D quasicrystal can have any finite number of letters. However, the minimum is two. The Fibonacci chain is the quintessential 1D quasicrystal.

Let us explicitly show the $1 \mathrm{D}$ quasicrystal construction that can be generated by an iterative process. We start with the two words, $W_{0}=L$, where $L$ equals the longer length in a concrete $1 \mathrm{D}$ quasicrystal made of distances between neighboring atoms, and $W_{1}=L S$, where $S$ is the shorter length. Let $W_{n}=W_{n-1} W_{n-2}$ be the concatenation of the previous two words. A explicit Fibonacci chain takes the form $W_{n}=L S L L S L S L L S L L S \ldots$... Alternatively, one can start with $W_{0}=L$ and apply the following substitution rules to iterate one word $W_{n}$ to the next $W_{n+1}$

$$
\begin{aligned}
& L \rightarrow L S \\
& S \rightarrow L .
\end{aligned}
$$

The following rules are also valid:

$$
\begin{aligned}
& L \rightarrow S L \\
& S \rightarrow L .
\end{aligned}
$$

The rules for creating the Fibonacci chain $W_{n}$ prohibit the formation of certain non-syntactically legal sub-words. For instance, there cannot be three consecutive $L$ 's appearing in $W_{n}$ nor can there be two $S$ 's next to each other. So $L L L$ and $S S$ break the code rules and are not valid Fibonacci chains. Furthermore, if one section of the code has $L L$ the next letter must be $S$. Likewise, an $S$ must always be followed by an $L$. After four iterations, for example, we can have two different legal words following one of the substitution rules above - 
LSL $L S L S L$ or LSLSLLSL. If we want to build the Fibonacci chain quasicrystal LSL $(L S$ or $S L)$ LSL directly, we have the freedom to choose the words in the middle by the cut-and-project method using the concepts of cut-window and empire-window [47]. This exemplifies the syntactical freedom and therefore the code theoretic nature of quasicrystal languages. These code theoretic substitution rules are of the same form as Fibonacci anyons fusion rules. And, as with the fusion rules, the substitution rules are exceedingly simple and possess spatiotemporal syntactical degrees of freedom to express higher level emergent physical meaning, such as the experimentally measured macro-scale ground states of real atomic quasicrystals.

A promising line of research is studying the role of quasicrystals in the representation theory of Lie groups [54-58]. Specifically, a quasicrystal is a cut-and-projection of a slice of a higher dimensional lattice that can correspond to the root vector polytope and lattice of a given Lie algebra. It is well known [59] that the weights of any representation of a Lie algebra are invariant under the action of the Weyl group - the point groups symmetry of the respective root lattice. The opportunity for using quasicrystalline codes for particle physics models lies in generalizations of the Weyl group to Coxeter (reflection) groups [60], which include the noncrystallographic groups - the symmetry point group of quasicrystals. The noncrystallographic groups, however, can be used to construct some of the Weyl groups [61]. There are two primary advantages to using the geometry and algebra associated with such projective transformations: (1) unlike the hyper-lattices from which they are transformed, quasicrystals are non-local and non-deterministic codes playing out dynamically and (2) they can exist in the more "physically realistic" lower dimensions in which physics seems to play out.

The two physical spatiotemporal codes presented in sub-sections (2.1) and (2.2) exemplify the idea of a code theoretical framework and they support the argument that there is a code theoretic principle linked with the representation theory of Lie groups, as the aforementioned codes are closely related to the representation theory of Lie groups. The anyonic topological codes are related to the representation theory of quantum groups - generalizations of Lie groups. Quasicrystalline codes are related to the root lattices of Lie algebras. The representation theory of Lie algebras is a foundational formalism used to describe symmetries in nature. In the next section we focus on the Poincaré Lie group to present the argument that the Planck scale quantum gravity regime is a physical code in the strictest sense of the term.

It should be noted that there is confusion around the interpretation of the results of string theory [62] and loop quantum gravity [63], mainly in the view that the quantum gravity regime is a chaotic quantum foam, wherein the challenge is to unravel the mechanisms that explain how order emerges from chaotic noise. Alternatively, one may instead focus on understanding that the quantum gravity scale should imply restrictions in spatiotemporal degrees of 
freedom to construct a new code theoretic discretized quantum field theory. The challenge then is how to recover the continuum symmetries at the large scale. If the code theoretic restrictions are merely arbitrary ad hoc restrictions, such as those we build into computer simulations in order to limit the number of the degrees of freedom to improve computational efficiency, we should not be able to recover experimentally verified gauge symmetries that correspond to the root vectors of higher dimensional Lie lattices and their associated algebras. Therefore, in line with the holographic principle and modern particle accelerator experiments, along with what our best fundamental theories show, we should expect to find a code at the Planck scale that correlates directly to these conserved gauge symmetry transformations. Pure randomness would recede to an antiquated conjecture and the non-deterministic and non-local behavior of physical codes would emerge to be a more physically realistic approach to explain the evolution of the continually broken-symmetry offequilibrium world we observe.

\section{Relativistic Quantum Fields and Representations of Lie groups}

The quantum field theory of the standard model of particle physics associates spin and mass quantum numbers with the Poincaré Lie group - the spin quantum number is associated with its Lorentz subgroup of rotations $S O(1,3)$ and the mass quantum number with its subgroup of translations. So the Poincaré group is needed for spin and mass. Charge (electric, color) is associated with internal (gauge) groups of symmetry [64-67]. Spacetime symmetries are represented thus by the Poincaré group that contains $S O(1,3)$ generators plus momentum generators. With focus on spin, the concept of relativistic fields is that they are finite representations of the Lorentz group. Classifications of these representations can be done with respect to their eigenvalues, in this case, spin quantum numbers. A general matrix $\Lambda_{\nu}^{\mu}$ of $S O(1,3)$ possesses the constraints $\operatorname{det} \Lambda= \pm 1$ and $\Lambda_{0}^{0} \geq 1$. This is the restricted Lorentz group $S O(1,3)$. It is a class of transformations whose finite elements are generated from infinitesimal transformations to the identity and, as a result, it is a Lie group [59]. The matrix can be written as

$$
\Lambda(w)=e^{\frac{1}{2} w^{\alpha \beta} \Sigma_{\alpha \beta}}
$$

where $w^{\alpha \beta}$ are infinitesimal parameters and $\Sigma_{\alpha \beta}$ are the generators ${ }^{4}$. We can rewrite the generators $\Sigma_{\alpha \beta}$ in terms of generators of two independent $S U(2)$ subalgebras. To do this, first we rewrite the generators in terms of angular momentum generators $M^{i}$ :

$$
M^{i}=\frac{1}{2} \varepsilon^{i j k} \Sigma_{j k}
$$

\footnotetext{
4 Spacetime indices like $\alpha, \beta, \mu, \nu$ run from 0 to 3 and the space indices $i, j, k$, run from 1 to 3 , with the convention for the metric tensor being $g_{\mu \nu}=\operatorname{diag}(1,-1,-1,-1)$.
} 
and the Lorentz boosts $N^{i}$ :

$$
N^{i}=\Sigma^{0 i} .
$$

Going then to a new basis with generators $J^{i}, G^{i}$ given by

$$
J^{i}=\frac{i}{2}\left(M^{i}+i N^{i}\right),
$$

and

$$
G^{i}=\frac{i}{2}\left(M^{i}-i N^{i}\right),
$$

one can check that these two generators $J^{i}$ and $G^{i}$ obey $S U(2)$ Lie algebra commutation relations:

$$
\begin{aligned}
{\left[J^{i}, J^{j}\right] } & =i \varepsilon^{i j k} J^{k}, \\
{\left[G^{i}, G^{j}\right] } & =i \varepsilon^{i j k} G^{k}, \\
{\left[J^{i}, G^{i}\right] } & =0 .
\end{aligned}
$$

Therefore, we can see from (9) that the Lorentz group representations can be written from these two complex $S U(2)$ representations with independent generators $J^{i}, G^{i} . S O(1,3)$ decomposes, as a direct sum, to

$$
S O(1,3)=S U(2)_{J} \oplus S U(2)_{G} .
$$

The need for complexification comes from the fact that the Lorentz group is non compact and allows one to work with the well known representation theory of $S U(2)$, which is compact. In particular, for each subalgebra $S U(2)$ there is a Casimir operator $J^{i} J^{i}, G^{i} G^{i}$, commuting with each element of the algebra and with eigenvalues $j(j+1), g(g+1), j, g \in 0, \frac{1}{2}, 1, \frac{3}{2}, 2, \ldots$ Being invariants, its eigenvalues are conserved and so they provide good quantum numbers to index the representations of $S O(1,3)$ by pairs $(j, g)$ with eigenvalues $j(j+1)$ and $g(g+1)$. The total spin of the representation $(j, g)$ is given by $s=j+g$ and its dimension by $\operatorname{dim}(j, g)=(2 j+1)(2 g+1)$.

For the Poincaré group as a whole we need to include the generators of spacetime translations $P^{\mu}$ together with the Lorentz generators $\Sigma_{\mu \nu}$ and the algebra is

$$
\begin{aligned}
{\left[P_{\mu}, P_{\nu}\right] } & =0 \\
{\left[\Sigma_{\alpha \beta}, P_{\mu}\right] } & =-i\left(g_{\alpha \mu} P_{\beta}-g_{\beta \mu} P_{\alpha}\right), \\
{\left[\Sigma_{\alpha \beta}, \Sigma_{\mu \nu}\right] } & =\left(g_{\alpha \nu} \Sigma_{\beta \mu}+g_{\beta \mu} \Sigma_{\alpha \nu}-g_{\alpha \mu} \Sigma_{\beta \nu}-g_{\beta \nu} \Sigma_{\alpha \mu}\right) .
\end{aligned}
$$

In this case there are two Casimir invariants, $P_{\mu} P^{\mu}$ and $W_{\mu} W^{\mu}$, where $W^{\mu}=$ $\frac{1}{2} \varepsilon^{\mu \nu \alpha \beta} P_{\nu} \Sigma_{\alpha \beta}$ is the Pauli-Lubanski four-vector operator. These operators act in the representations with eigenvalues $m^{2}$ and $-m^{2} s(s+1)$, respectively. According to the well known Wigner classification [67], relativistic particles are associated with the Poincaré group and the relativistic fields with the Lorentz group.

Let us consider several examples of irreducible representations in the case of the Lorentz group: 
- The representation $(0,0)$, with spin 0 , is the representation of the scalar field.

- The representation $\left(\frac{1}{2}, 0\right)$ corresponds to left-handed fermions. The dimension is 2 , so the generators are $2 \times 2$ matrices, which act on objects with 2 complex components, the Weyl spinors, and can describe, for example, massless neutrinos.

- The representation $\left(0, \frac{1}{2}\right)$ is analogous, with the spinors being right-handed.

- The representation $\left(\frac{1}{2}, 0\right) \oplus\left(0, \frac{1}{2}\right)$ has dimension 4 and can describe the electron and positron.

- The representation $\left(\frac{1}{2}, \frac{1}{2}\right)$ has dimension 4 , where one is the degree of freedom associated with spin 0 , a scalar field, and 3 are from spin 1, a vector field. The two fields are the components of the 4 vector field used to describe bosons such as the photon. Interestingly, the framework of gauge theory makes use of this large gauge symmetry object to describe the two degrees of freedom of the photon. This is because the decomposition (10) is not fully relativistic, which results in some representations having more degrees of freedom than are physically realistic. The elimination of these spurious degrees of freedom is part of the motivation and power of gauge symmetry unification physics [64-66].

So far, just spin $0, \frac{1}{2}$ and 1 have experimental support and mathematical consistency with local relativistic quantum field theory [68]. Spin $\frac{3}{2}$ and spin 2 are expected to be physically realistic when gravity is included in the picture, but for now they appear only in theoretical extensions of the standard model. For higher spin representations, on the other hand, there is little hope for experimental evidence.

With this compact description of representation theory of the Poincaré group and especially its $S O(1,3)$ subgroup, we are prepared to put forth our argument. Restricting our attention to one $S U(2)$ in (10) we realize that just spin $0, \frac{1}{2}$ and 1 appear in the experimentally validated predictions of the standard model of particle physics and we can point out theoretic support for this restriction with the Weinberg-Witten theorem [68]. This realization that nature needs only the lower dimensional representations from the infinite possibilities in one $S U(2)$ is important phenomenology that indicates nature is code theoretic. Like in the physical codes discussed in Section (2), in this representation theory constrained by physics, we have a few letters labeled by the lower spins (specific representations), fusion rules given by the recoupling theory of $S U(2)$, and the freedom in how these representations can be coupled to express physical meaning, in this case, the relativistic quantum fields observed in nature. The challenge consists in implementing the code theoretic principle in a mathematically consistent way so that we can predict the physically realistic representations and how they are coupling in a physically realistic code theoretic manner. We can elucidate clues from the so-called quantization of Lie groups at roots of unity. Moreover, we can use two quantizations of $S U(2)$ at roots of unity in order to address the quantum Poincaré group. With 
this "quantization" the representation theory of spacetime symmetry can be restricted to a few sets of representations with well defined fusion rules.

\subsection{Quantum $S O(1,3)$ at the 5 th root of unity}

With the understanding of elementary particles as irreducible representations of the Poincaré group, it is natural to formulate a quantum field theory based on a quantum Poincaré group, i.e., on quantized spacetime [35,69-71]. Quantum groups are deformations on Hopf algebras, which allow generalizations of Lie groups and Lie algebras [35,72-74]. These deformations on Hopf algebras depend on a deformation parameter $q$. When $q$ is a real parameter, the representation theory is the same as the classical group. If we allow $q$ to have arbitrary complex values, the $q$-deformed universal enveloping algebra becomes complex with non-unitary representations. However, in the special case where $q$ is a complex root of unity, ${ }^{5}$ there are new types of representations helpful in achieving the desired restrictions on the classical representations discussed in Section 3. To fulfill this objective we require a consistent theory that allows for only the aforementioned physically realistic representations that have been experimentally confirmed to appear in the fusion rules. For example, for $q$, a complex root of unity, only a specific set of representations, delimited by the specific root, are irreducible and unitary.

For $S U(2)_{q}$, where $q=e^{\frac{2 i \pi}{5}}$ is the 5 th root of unity, it is possible to find unitary irreducible representations, which agree with the classical ones that are physically realistic; these are the lower dimensional ones discussed in the previous section, spin 0 , spin $\frac{1}{2}$, spin 1 and spin $\frac{3}{2}$, the other ones being indecomposable and non unitary. To show this we will focus on just $S U(2)_{J}$ in the decomposition (10). The second, $S U(2)_{G}$, is analogous. We can define raising and lowering operators as in the theory of angular momentum from (7)

$$
J_{ \pm}=J_{1} \pm i J_{2}
$$

with

$$
\begin{aligned}
{\left[J_{3}, J_{ \pm}\right] } & = \pm J_{ \pm} \\
{\left[J_{+}, J_{-}\right] } & =2 J_{3} .
\end{aligned}
$$

We can then introduce the deformation generator

$$
\mathcal{J}=q^{J_{3}} \text {. }
$$

The $S U(2)_{q}$ algebra, which is over the complex numbers, is generated by the three operators $\mathcal{J}, J_{ \pm}$

$$
\begin{aligned}
{\left[J_{+}, J_{-}\right] } & =2 \frac{\mathcal{J}-\mathcal{J}^{-1}}{q-q^{-1}} \\
\mathcal{J} J_{ \pm} \mathcal{J}^{-1} & =q^{ \pm 2} J_{ \pm}
\end{aligned}
$$

${ }^{5}$ In the case of a complex root of unity $q$, the q-deformed universal enveloping algebra of $S U(2)$ for example, $\mathcal{U}_{q}(S U(2))$ or for short $S U(2)_{q}$ is a modular fusion category [37]. 
where the limit $q \rightarrow 1$ reproduces the classical algebra ${ }^{6}$ (13). There are significant new features of quantum group symmetry whose role in gauge and code theory we will explore in future work. For example, there are more invariants than the Casimir invariant (more quantum numbers) and the co-multiplication is not commutative, allowing braid theory to be employed [35].

Here we focus on the restriction we have achieved with the allowed irreducible representations. This relative representation theory is well understood $[35,75]$. There are two types of representations, the so-called nilpotent representations, for which the classical analogous irreducible representations are also well defined, and the cyclic representations, which do not have a classical analogue. We will focus on the nilpotent representations, which have a classical analogue. However, just the ones in a specific range of spins are admissible. For this specific situation of the 5 th root of unity, ${ }^{7}$ the admissible spins are $j=0, \frac{1}{2}, 1, \frac{3}{2}$, their quantum dimension is given by $d_{j}^{q}=\sin \left(\frac{\pi(2 j+1)}{5}\right) / \sin \left(\frac{\pi}{5}\right)$ and its composition of representations follow the following fusion rules:

$$
\begin{aligned}
0 \otimes j & =j \\
\frac{3}{2} \otimes j & =\frac{3}{2}-j \\
\frac{1}{2} \otimes \frac{1}{2} & =0 \oplus 1 \\
\frac{1}{2} \otimes 1 & =\frac{1}{2} \oplus \frac{3}{2} \\
1 \otimes 1 & =0 \oplus 1 .
\end{aligned}
$$

This fusion algebra together with the equivalent one for the second $S U(2)_{G}$ in the decomposition of the Lorentz group gives us a quantization of this group at the 5 th root of unity at least in this non-relativistic sector ${ }^{8}$ with representations $(j, g)$ limited to $j, g \in 0, \frac{1}{2}, 1, \frac{3}{2}$ and the fusion rules in (16). Of these, the only representations that have not been observed yet are the ones involving $\frac{3}{2}$. One important result we can highlight here is that the physics of the fundamental building blocks - spin $\frac{1}{2}$ and spin 1 - does not necessarily distinguish the classical algebra (13) from the quantum one (15). This can be seen using an explicit matrix representation with the usual Pauli matrices $\sigma_{i}$. For the classical algebra this is straightforward, and for the quantum one we can write for example the right side of the first equation in (15) as

\footnotetext{
${ }^{6}$ For arbitrarily large roots of unity, we can write $q$ using a small complex number $\epsilon$, $q=e^{\epsilon}$ and we can formally expand to first order $\mathcal{J}=q^{J_{3}}=1+\epsilon J_{3}+O\left(\epsilon^{2}\right)$ and write $q=1+\epsilon$. In doing so we can recover (13). Accordingly, with the 5th root of unity, we avoid the classical situation with its non-physically realistic infinite representations.

7 See for example chapter 6 in reference [35].

8 As mentioned earlier in this section, this sector is relevant in describing the spin degree of freedom. The implication is that there are spurious degrees of freedom in the ordinary gauge field description, which are important in the usual construction of gauge theories, such as the spin 0 present in $\left(\frac{1}{2}, \frac{1}{2}\right)$. Here, the spin 0 is implied by the fusion rule. From the point of view of the anyonic topological code discussed in Section (2), this allows for the desired freedom in the code.
} 
$\left(e^{\frac{2 \pi i}{5} \sigma_{3}}-e^{-\frac{2 \pi i}{5} \sigma_{3}}\right) /\left(e^{\frac{2 \pi i}{5}}-e^{-\frac{2 \pi i}{5}}\right)$ and then one can show that this is equal to $\sigma_{3}$. The same can be shown for spin 1 with a rescaling of $J_{+}, J_{-}$. The two symmetries are almost equivalent at the level of spin $\frac{1}{2}$ and spin 1 , but the 5 th root of unity quantization symmetry avoids the non-physical higher dimensional representations. The hope is that a full quantization of the Lorentz group at the 5th root of unity will give us the correct representations that are experimentally observed and will allow predictions of possible new ones. In other words, we expect the full quantization of the Poincaré group to give us the correct standard model masses and possibly new masses beyond standard model physics. This full quantization with a deformation parameter being a complex root of unity was done in the context of $\kappa$-deformed symmetries [76]. The deformation parameter's restriction to the 5 th root of unity is under investigation.

Thus far, we have discussed spacetime symmetries, but this same 5 th root of unity quantization of $S U(2)$ can help us understand restrictions on the representation theory of charge space. The weak charge is described by $S U(2)$ and the color charge by $S U(3)$ Lie groups. Following the well known Cartan-Weyl basis description of Lie algebras, representation theory of $S U(3)$ can be understood in terms of $S U(2)$ sub-group representations [77]. The $S U(2)_{q}$ at the 5 th root of unity restricts those representations to the ones that are experimentally verified - the lower dimensional "fundamental" and "adjoint" representations. We can emphasize that yet another motivation for these restrictions imposed by the 5th root of unity quantization is given by the covariant loop quantum gravity quantization of general relativity. The Hilbert space that results from this quantization is described by spin network quantum amplitudes, a spin network being essentially an interaction network of $S U(2)$ representations [63]. Transition amplitudes for quantum geometries can be computed taking into account only the representations that have a counterpart in the field/particle matter content in a consistent way with the aforementioned 5th root of unity quantization. These results will be presented in an upcoming paper.

It is remarkable that we can recover the quantum symmetry $S U(2)_{q}$ at the 5 th root of unity in different models of quantum gravity and particle physics unification. In these approaches, $q$ is considered to be an arbitrary complex root of unity, which, of course, means the 5 th root of unity solution ${ }^{9}$ is included and supports our discussion here. For example, $S U(2)_{q}$ appears in the quantization of string theory on a group manifold [78], with a focus on the $S U(2)$ group, and string-net models of gauge field emergence [79]. It also appears throughout the so-called quantum group conformal field theory duality [43] and in topological quantum field theory [80,81]. In quantum gravity it defines a special base for the Hilbert space of loop quantum gravity, which is one of the promising ways

\footnotetext{
9 Since in our approach we use the Fibonacci anyon theory and the fusion rules of spin 1 (with quantum dimension equal to the golden ratio $\phi$ ), which resemble the known Fibonacci sequence fusion rules, also connected with properties of the golden ratio, we can simply call this solution phi-field.
} 
of achieving quantum spin networks linked with the cosmological constant [63, 82-85].

\section{Conclusions}

In this paper we have presented several results supporting the idea that there is a code theoretic principle correlated with the physical realization of representations of Lie groups, in particular the Poincaré group. This indicates that quantizations of spacetime must respect a special kind of quantum symmetry implemented as a code made of a small set of representations of its symmetry group, each with specific fusion rules and syntactical degrees of freedom that express physical meaning in the form of quantum fields at large scales. In line with quantum information and digital physics principles applied to spacetime, the code theoretic framework is a novel and logical approach with potential to bring new advances in quantum gravity and unification physics. The usual manner in which relativistic theory relates mass, energy and geometry, together with the conceptual manner in which quantum mechanics integrates information in the description of fundamental physical systems, can be improved by including computations in a code theoretic framework.

In this study we presented one initial quantization of the Lorentz subgroup of the Poincaré group at the 5th root of unity, at the level of its Lie algebra, by using the usual decomposition in terms of two complex $S U(2)$ Lie algebras. The 5 th root of unity quantization provides the spin quantum numbers needed to describe the known elementary particles following the usual Wigner classification of relativistic fields, which maps these mathematical objects the representations of the symmetry group - to the physical fields. With the classical symmetry there are infinite representations and so one would expect infinite types of fields, which are not observed. The 5th root of unity quantization representations match the observed fields associated with spin 1 and spin $\frac{1}{2}$, being in this case almost equivalent to the classical ones. This also emphasizes the importance of the "fundamental" and "adjoint" representations of the charge groups $S U(2)$ and $S U(3)$. The irreducible representations are the lower dimensional ones that appear in the few tensor products in the fusion rules (16).

Furthermore, we point out the fundamental importance of a full quantization of the Poincaré group with a special emphasis on the 5th root of unity. We stress another development, which is the subject of our ongoing work: the implication of quantum symmetry to the internal group of symmetries associated with charge via the elimination of spurious degrees of freedom, which relate to the spin representations that are not fully relativistic, while at the same time giving the correct observed spectrum. The root lattices, which appear in representation theories of Lie groups, allow one to build quasicrystalline codes at lower dimensions where the building blocks are representations of these groups, in agreement with the code theoretic principle discussed herein - a quasicrystalline spin network. 
This study opens many directions of research, one of which is to investigate in detail the unitary irreducible representations of the full quantum Poincare group for complex q at a 5 th root of unity. Moreover, since the root lattices of the unification groups $S U(5), S O(12)$ and $E 8$, which encode information of representations of these Lie groups, can be projected to the quasicrystals associated with the non crystallographic Coxeter groups $H 2, H 3$ and $H 4$, respectively $[57,55]$, one can investigate the quasicrystalline representations of these unification groups and their correlation with the quantization described here. In a quantum gravity context, a deeper analysis of the spin network transition amplitudes is needed, in particular the geometric interpretation of the spin network constrained to the 5th root of unity quantization.

\section{Acknowledgements}

We would like to thank Carlos Castro Perelman and Sinziana Paduroiu for reviewing the manuscript and making useful suggestions.

\section{References}

1. 't Hooft, G.: Dimensional reduction in quantum gravity, Conf. Proc. C 930308, 284 (1993)

2. Susskind, L.: The World as a hologram, J. Math. Phys. 36, 6377 (1995)

3. Maggiore, M.: Quantum groups, gravity, and the generalized uncertainty principle, Phys. Rev. D 49, 5182 (1994)

4. Kowalski-Glikman, J.: Observer independent quantum of mass, Phys. Lett. A 286, 391 (2001)

5. Amelino-Camelia, G.: Relativity in space-times with short distance structure governed by an observer independent (Planckian) length scale, Int. J. Mod. Phys. D 11, 35 (2002)

6. Tawfik, A. N., Diab, A. M.: Review on Generalized Uncertainty Principle, Rept. Prog. Phys. 78, 126001 (2015)

7. Hu, Jinwen: The Non-Lorentz Transformation Corresponding to the Symmetry of Inertial Systems and a Possible Way to the Quantization of Time-Space, Physics Essays 30, no. 3: 322-327. (2017).

8. Liberati, S., Maccione, L.: Lorentz Violation: Motivation and new constraints, Ann. Rev. Nucl. Part. Sci. 59:245-267, (2009)

9. Ali, A. F.: Minimal Length in Quantum Gravity, Equivalence Principle and Holographic Entropy Bound, Class. Quant. Grav. 28:065013, (2011)

10. Penrose, R.: Fashion, Faith, and Fantasy in the New Physics of the Universe, Princeton University Press (2016)

11. Rovelli, C.: Reality Is Not What It Seems: The Journey to Quantum Gravity, Riverhead Books; 1st edition (2017)

12. Smolin, L.: The Trouble With Physics, Mariner Books; Reprint edition (2007).

13. Witten, E.: Symmetry and Emergence, Nature Physics 14, 116119 (2018)

14. Irwin, K.: The Code-Theoretic Axiom, FQXI Essay Contest (2017), URL: http://fqxi.org/community/forum/topic/2901. Accessed 01 May 2018

15. Conway, J., Kochen, S.: The free will theorem, Foundations of Physics 36 (10), 1441-1473 (2006)

16. Conway, J. H., Kochen, S.: The strong free will theorem, Notices of the AMS56(2),226$232(2009)$

17. Maldacena J., Susskind, L.: Cool horizons for entangled black holes, Fortsch. Phys. 61, $781(2013)$ 
18. Amaral, M. M., Irwin, K., Fang, F., Aschheim, R.: Quantum walk on a spin network. In: Proceedings of the Fourth International Conference on the Nature and Ontology of Spacetime, 30 May - 2 June 2016, Golden Sands, Varna, Bulgaria. Minkowski Institute Press, Montreal (Quebec, Canada). (2017) arXiv:1602.07653 [hep-th].

19. Shenker, S. H., Stanford, D.: Black holes and the butterfly effect, JHEP 1403, 067 (2014)

20. Van Raamsdonk, M.: Building up spacetime with quantum entanglement, Gen. Rel. Grav. 42, 2323 (2010); Int. J. Mod. Phys. D 19, 2429 (2010)

21. Ryu, S., Takayanagi, T.: Holographic derivation of entanglement entropy from AdS/CFT, Phys. Rev. Lett. 96, 181602 (2006)

22. 't Hooft, G.: The Cellular Automaton Interpretation of Quantum Mechanics, arXiv:1405.1548 [quant-ph] (2014). Accessed 01 May 2018

23. Aschheim, R.: Hacking reality code. FQXI Essay Contest 2010-2011 - Is Reality Digital or Analog? (2011). URL: http://fqxi.org/community/forum/topic/929. Accessed 01 May 2018

24. Wheeler, J. A.: Hermann Weyl and the unity of knowledge, American Scientist 74: 366-375, (1986)

25. Wheeler, J. A.: Information, physics, quantum: The search for links. In: Complexity, Entropy, and the Physics of Information. Redwood City, CA: Addison-Wesley. Cited in DJ Chalmers,(1995) Facing up to the Hard Problem of Consciousness, Journal of Consciousness Studies 2.3: 200-19 (1990)

26. Tegmark, M.: Is the theory of everything merely the ultimate ensemble theory?, Annals of Physics 270.1: 1-51 (1998)

27. Tegmark, M.: The mathematical universe, Foundations of Physics 38.2: 101-150 (2008)

28. Miller, D. B., Fredkin, E.: Two-state, Reversible, Universal Cellular Automata In Three Dimensions, Proceedings of the ACM Computing Frontiers Conference, Ischia, (2005)

29. Fredkin, E.: Digital Mechanics, Physica D 45, 254-270 (1990)

30. Fredkin, E.: An Introduction to Digital Philosophy, International Journal of Theoretical Physics, Vol. 42, No. 2, 189-247 (2003)

31. Wolfram, S.: A New Kind Of Science, Wolfram Media, Inc., (2002)

32. Susskind, L.: Dear Qubitzers, GR=QM, (2017) arXiv:1708.03040 [hep-th]. Accessed 01 May 2018

33. Aschheim, R.: SpinFoam with topologically encoded tetrad on trivalent spin networks, Proceedings, International Conference on Non-perturbative / background independent quantum gravity (Loops 11): Madrid, Spain, May 23-28, slide 10, 24 may 2011, 19h10, Madrid (2011)

34. Irwin, K.: Toward a unification of physics and number theory, (2017).

https://www.researchgate.net/publication/314209738

35. Biedenharn, L. C., Lohe, M. A.: Quantum group symmetry and q-tensor algebras, World Scientific (1995)

36. Yan, S. Y.: An Introduction to Formal Languages and Machine Computation, World Scientific Pub Co Inc (1996)

37. Wang, Z.: Topological Quantum Computation, Number 112. American Mathematical Soc., (2010)

38. Pachos, J. K.: Introduction to Topological Quantum Computation, Cambridge University Press, (2012)

39. Wilczek, F.: Quantum Mechanics of Fractional-Spin Particles, Physical Review Letters. 49 (14): 957-959. (1982).

40. Slingerland, J. K., Bais, F. A.: Quantum groups and nonAbelian braiding in quantum Hall systems, Nucl. Phys. B 612, 229 (2001)

41. Kitaev, A. Y., Shen, A. H., Vyalyi, M. N.: Classical and Quantum Computation, American Mathematical Society, Providence (1999)

42. Freedman, M., Larsen, M., Wang, Z.: A modular functor which is universal for quantum computation, Commun. Math. Phys. 227: 605. (2002)

43. Alvarez-Gaume, L., Sierra, G., Gomez, C.: Topics In Conformal Field Theory, In: Brink, L. (ed.) et al.: Physics and mathematics of strings, 16-184 and CERN Geneva - TH. 5540 $169 \mathrm{p}(1990)$

44. Yichen Hu, Kane, C. L.: Fibonacci Topological Superconductor, Phys. Rev. Lett. 120, 066801 (2018) 
45. Schrödinger, E.: What Is Life? The Physical Aspect of the Living Cell, Cambridge University Press, (1967)

46. Senechal, M. L.: Quasicrystals and Geometry, Cambridge University Press, (1995)

47. Fang, F., Hammock, D., Irwin, K.: Methods for Calculating Empires in Quasicrystals, Crystals, 7(10), 304. (2017)

48. Baake, M., Grimm, U.: Aperiodic Order, Cambridge University Press, (2013)

49. Patera, J.: "Quasicrystals and discrete geometry", volume 10. American Mathematical Soc., (1998)

50. Fang, F., Kovacs, J., Sadler, G., Irwin, K.: An icosahedral quasicrystal as a packing of regular tetrahedra, ACTA Physica Polonica A 126(2), 458-460 (2014)

51. Shechtman, D., Blech, I., Gratias, D., Cahn, J. W.: Metallic phase with long-range orientational order and no translational symmetry, Physical Review Letters, 53(20):1951, (1984)

52. Elser, V., Sloane, N. J. A.: A highly symmetric four-dimensional quasicrystal, J. Phys. A, 20:6161-6168, (1987)

53. Barber, E. M.: Aperiodic structures in condensed matter, CRC Press, Taylor \& Francis Group, (2009)

54. Moody, R. V., Patera, J.: Quasicrystals and icosians, J. Phys. A 26, 2829 (1993)

55. Chen, L., Moody, R. V., Patera, J.: Non-crystallographic root systems, In: Quasicrystals and discrete geometry. Fields Inst. Monogr, (10), (1995)

56. Koca, M., Koca, R., Al-Barwani, M.: Noncrystallographic Coxeter group H4 in E8, J.Phys.,A34,11201 (2001)

57. Koca, M., Koca, N. O., Koca, R.: Quaternionic roots of E8 related Coxeter graphs and quasicrystals, Turk.J.Phys.,22,421 (1998)

58. Sanchez, R., Grau, R.: A novel Lie algebra of the genetic code over the Galois field of four DNA bases, Math Biosci. 2006 Jul;202(1):156-74. Epub (2006)

59. Gilmore, R.: Lie groups, Lie algebras, and some of their applications, Dover Publications, INC. Dover edition (2005)

60. Geck, M., Pfeiffer, G.: Characters of Finite Coxeter Groups and Iwahori-Hecke Algebras, Oxford University Press; 1 edition (2000)

61. Moody, R. V., Morita, J.: Discretization of SU(2) and the orthogonal group using icosahedral symmetries and the golden numbers, Communications in Algebra, 46:6, 2510-2533, (2017)

62. Green, M. B., Schwarz, J. H., Witten, E.: Superstring Theory, Vol. I and Vol. II, Cambridge University Press (1988)

63. Rovelli, C., Vidotto, F.: Covariant Loop Quantum Gravity, Cambridge University Press 1 edition, (2014)

64. Zee. A. Quantum Field Theory in a Nutshell, Second edition. Princeton University Press. (2010)

65. Weinberg, S.: The Quantum Theory Of Fields Vol 1 Foundations, Cambridge University Press; 1st Edition edition (2005)

66. Franco, D. H. T., Helayël-Neto, J. A.: Lecture notes on the introduction to gauge theory course. CBPF, Brazil (2010)

67. Wigner, E. P.: On unitary representations of the inhomogeneous Lorentz group, Annals of Mathematics, 40 (1): 149-204 (1939)

68. Weinberg, S., Witten, E.: Limits on Massless Particles, Phys. Lett. 96B, 59 (1980)

69. Steinacker, H.: Finite dimensional unitary representations of quantum anti-de Sitter groups at roots of unity, Commun. Math. Phys. 192, 687 (1998)

70. Finkelstein, R. J.: SLq(2) extension of the standard model, Phys. Rev. D 89, no. 12, 125020 (2014)

71. Bojowald, M., Paily G. M., Deformed General Relativity, Phys. Rev. D 87, no. 4, 044044 (2013). [arXiv:1212.4773 [gr-qc]].

72. Drinfeld, V. Quantum Groups. Proceedings of the International Congress of Mathematicians, Berkeley, (1986). A.M. Gleason (ed.), 798, AMS, Providence, RI.

73. Faddeev, L. D., Reshetikhin, N. Y., Takhtajan, L. A.: Quantization of Lie Groups and Lie Algebras, Algebra Anal. 1. 178 (1989)

74. Jimbo, M,: A q-Difference Analogue of $U(g)$ and the Yang-Baxter Equation, Lett. Math. Phys 10, 63 (1985) 
75. Majid, S.: Foundations of quantum group theory, Cambridge University Press (1995)

76. Lukierski, J.: Kappa-Deformations: Historical Developments and Recent Results, J. Phys. Conf. Ser. 804, no. 1, 012028 (2017)

77. Georgi, H.: Lie algebras in particle physics, from isospin to unified theories, Westview Press (1999)

78. Gepner, D., Witten, E.: String Theory on Group Manifolds, Nucl. Phys. B 278, 493 (1986)

79. Levin, M. A., Wen, X. G.: String net condensation: A Physical mechanism for topological phases, Phys. Rev. B 71, 045110 (2005)

80. Witten, E.: Topological Quantum Field Theory, Commun. Math. Phys. 117, 353 (1988)

81. Witten, E. Quantum Field Theory and the Jones Polynomial, Commun. Math. Phys. 121, 351 (1989)

82. Turaev, V. G., Viro, O. Y.: State sum invariants of 3 manifolds and quantum $6 \mathrm{j}$ symbols, Topology 31, 865 (1992).

83. Crane, L., Yetter, D. N.: A categorical construction of 4D TQFTs, Quantum Topology. 120-130. (1993)

84. Crane, L., Kauffman, L. H., Yetter, D. N.: State-Sum Invariants of 4-Manifolds I, Journal of Knot Theory and Its Ramifications 06:02, 177-234. (1997)

85. Dittrich, B., Geiller, M.: Quantum gravity kinematics from extended TQFTs, New J. Phys. 19, no. 1, 013003 (2017) 